\title{
3D Elasto-Plastic Stress Analysis by the Method of Arbitrary Lines*
}

\author{
Ken KAMINISHI** and Ryuma ANDO**
}

\begin{abstract}
The method of arbitrary lines (MAL) constitutes a general dimensional reduction methodology for elliptic boundary value problems (BVP) in arbitrary two ${ }^{ \pm}$and threedimensional domains by solving systems of one-dimensional boundary value ordinary differential equations (ODEs). It has been already applied to two-dimensional problem, and the good results have been reported. In this work, we consider the extension of the MAL to three-dimensional elasto-plastic stress analysis. We first give the MAL formulation of three-dimensional elasto-plastic problems. Although the MAL formulation is derived from the principle of three-dimensional increment virtual work as well as the finite element method (FEM), the MAL is different from FEM in that displacement increment and virtual displacement increment are expressed continuous, functions along one direction and shape-functions along other two directions. Substituting displacement increment and virtual displacement increment into the principle of three-dimensional increment virtual work, we have a system of ODEs. The threedimensional elasto-plastic analysis of BGA model, which was a method of the solder joints of electronic component, was carried out. As results, it was confirmed that to solve 3D elasto-plastic problem at the good accuracy was possible by the MAL.
\end{abstract}

Key Words : Computational Mechanics, Numerical Analysis, Plasticity, Nonlinear Problem, Three Dimentional Analysis, Method of Arbitrary Lines

\section{Introduction}

The Method of Arbitrary lines (MAL) is a new general-purpose numerical analysis method which employs a technique of reducing a governing equation to a system of ordinary differential equations. A high level of accuracy can be expected by this analysis for various problems, since it does not require discretization in some unidirection. It has already been applied to 2D (two-dimensional) elastic, elastoplastic problems and 3D (three-dimensional) elastic problems, and good results have been reported ${ }^{(1)-(4)}$. In this work we consider the extension of the MAL to 3D elastoplastic problems. Because 2D elastoplastic analysis method proposed previous work ${ }^{(3)}$ is the direct method which solves the nonlinear equations by linearizing, it is anticipated that the considerable calculation time is

* Received 16th May, 2002. Japanese original: Trans, Jpn. Soc. Mech. Eng., Vol. 67, No.654, A (2001), pp. 244-251, (Received 11th April, 2000)

** Yamaguchi University, Department of Mechanical Engineering, 2-16-1 Tokiwadai, Ube, Yamaguchi 755-8611, Japan. E-mail: kaminisi@yamaguchi-u. ac.jp needed when the technique is expanded as it is in the $3 \mathrm{D}$ problem. Therefore, we first derive the principle of discrete virtual work by MAL based on the iterative algorithm for 3D elastoplastic problems and propose a method for solving the discrete systems. Furthermore, the effectiveness of the $3 \mathrm{D}$ elastoplastic analysis by MAL is examined by comparing the MAL solutions with the FEM ones.

\section{The Principle of Discrete Virtual Work by} the 3D Elastoplastic MAL

The principle of the incremental virtual work for $3 \mathrm{D}$ small deformation elastoplastic problem is

$$
\begin{aligned}
& \iiint_{\Omega}\left\{D_{11} \frac{\partial d u}{\partial x} \frac{\partial d \delta u}{\partial x}+D_{12} \frac{\partial d v}{\partial y} \frac{\partial d \delta u}{\partial x}\right. \\
& \quad+D_{13} \frac{\partial d w}{\partial z} \frac{\partial d \delta u}{\partial x}+D_{21} \frac{\partial d u}{\partial x} \frac{\partial d \delta v}{\partial y} \\
& \quad+D_{22} \frac{\partial d v}{\partial y} \frac{\partial d \delta v}{\partial y}+D_{23} \frac{\partial d w}{\partial z} \frac{\partial d \delta v}{\partial y} \\
& \quad+D_{31} \frac{\partial d u}{\partial x} \frac{\partial d \delta w}{\partial z}+D_{32} \frac{\partial d v}{\partial y} \frac{\partial d \delta w}{\partial z} \\
& \quad+D_{33} \frac{\partial d w}{\partial z} \frac{\partial d \delta w}{\partial z} \\
& \quad+D_{44}\left(\frac{\partial d u}{\partial y}+\frac{\partial d v}{\partial x}\right)\left(\frac{\partial d \delta u}{\partial y}+\frac{\partial d \delta v}{\partial x}\right)
\end{aligned}
$$




$$
\begin{aligned}
& +D_{55}\left(\frac{\partial d v}{\partial z}+\frac{\partial d w}{\partial y}\right)\left(\frac{\partial d \delta v}{\partial z}+\frac{\partial d \delta w}{\partial y}\right) \\
& \left.+D_{66}\left(\frac{\partial d w}{\partial x}+\frac{\partial d u}{\partial z}\right)\left(\frac{\partial d \delta w}{\partial x}+\frac{\partial d \delta u}{\partial z}\right)\right\} d x d y d z \\
& =\iiint_{\Omega}\left(d f_{x} d \delta u+d f_{y} d \delta v+d f_{z} d \delta w\right) d x d y d z \\
& +\iint_{\Gamma_{N}}\left(d h_{x} d \delta u+d h_{y} d \delta v+d h_{z} d \delta w\right) d s \\
& + \text { Res. }
\end{aligned}
$$

$d u, d v, d w$ are the incremental displacements of $x, y$, $z$ direction, respectively, $d \delta u, d \delta v, d \delta w$ are the corresponding incremental virtual displacements $d f_{x}, d f_{y}$, $d f_{z}$ are corresponding increment body forces and $d h_{x}$, $d h_{y}, d h_{z}$ are corresponding increment tractions. Res in right-hand side is residual energy expressed as follows.

$$
\begin{aligned}
& \text { Res }=\iiint_{\Omega}\left\{\frac{\partial d \delta u}{\partial x} d \sigma_{x}^{I}+\frac{\partial d \delta v}{\partial y} d \sigma_{y}^{I}+\frac{\partial d \delta w}{\partial z} d \sigma_{z}^{I}\right. \\
& +\left(\frac{\partial d \delta u}{\partial y}+\frac{\partial d \delta v}{\partial x}\right) d \tau_{x y}^{I}+\left(\frac{\partial d \delta v}{\partial z}+\frac{\partial d \delta w}{\partial y}\right) d \tau_{y z}^{I} \\
& \left.\quad+\left(\frac{\partial d \delta w}{\partial x}+\frac{\partial d \delta u}{\partial z}\right) d \tau_{z x}^{I}\right\} d x d y d z .
\end{aligned}
$$

Where

$$
d \sigma^{I}=\boldsymbol{D} d \varepsilon^{p}
$$

$\boldsymbol{D}$ is elastic stress-strain matrix and $d \varepsilon^{p}$ is plastic strain increment.

In MAL, it is considered that element $\Omega_{i}$ constituted by the arbitrary curves is an image by $\Phi_{i}$ of master element $\widehat{\Omega}$ as shown in Fig. 1. Using $\boldsymbol{X}$ without discretization in the $\zeta$ and shape function $Q$ expressed by $\xi$, $\eta$, displacement increment of the $x, y$ and $z$ direction in $\Omega_{i}$ will be defined as follows.

$$
\begin{aligned}
& \left.d u\right|_{\Omega_{i}}=d u\left(\Phi_{i}(\xi, \eta, \zeta)\right)=\sum_{j}^{p} X_{x j}^{i}(\zeta)^{T} Q_{j}(\xi, \eta) \\
& \quad=\boldsymbol{X}_{x}^{i T} \boldsymbol{Q} \\
& \left.d v\right|_{\Omega_{i}}=d v\left(\Phi_{i}(\xi, \eta, \zeta)\right)=\sum_{j}^{p} X_{y j}^{i}(\zeta)^{T} Q_{j}(\xi, \eta) \\
& \quad=\boldsymbol{X}_{y}^{i T} \boldsymbol{Q} \\
& \left.d w\right|_{\Omega_{i}}=d w\left(\Phi_{i}(\xi, \eta, \zeta)\right)=\sum_{j}^{p} X_{z j}^{i}(\zeta)^{T} Q_{j}(\xi, \eta) \\
& =\boldsymbol{X}_{z}^{i T} \boldsymbol{Q}
\end{aligned}
$$

Where $p$ is the number of lines which constitute $\Omega_{i}$, and virtual displacement increment will be also defined as follows.
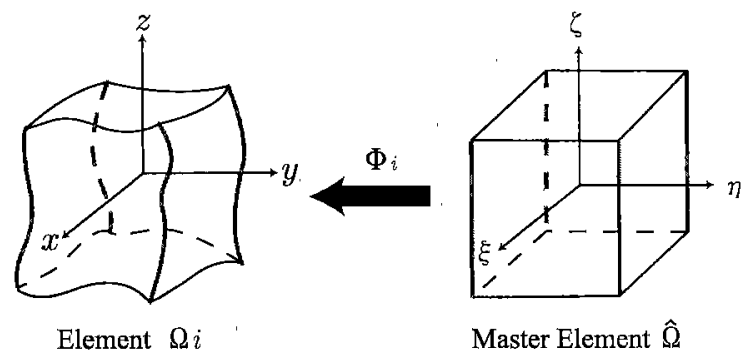

Fig. 1 Three dimensional mapping for the MAL

$$
\begin{aligned}
& \left.d \delta u\right|_{\Omega_{i}}=d \delta u\left(\Phi_{i}(\xi, \eta, \zeta)\right)=\sum_{j}^{p} \Psi_{x j}^{i}(\zeta)^{T} Q_{j}(\xi, \eta) \\
& \quad=\Psi_{x}^{i T} \boldsymbol{Q} \\
& \left.d \delta v\right|_{\Omega_{i}}=d \delta v\left(\Phi_{i}(\xi, \eta, \zeta)\right)=\sum_{j}^{p} \Psi_{y j}^{i}(\zeta)^{T} Q_{j}(\xi, \eta) \\
& \quad=\boldsymbol{\Psi}_{3}^{i T} \boldsymbol{Q} \\
& \left.d \delta w\right|_{\Omega_{i}}=d \delta w\left(\Phi_{i}(\xi, \eta, \zeta)\right)=\sum_{j}^{p} \Psi_{z j}^{i}(\zeta)^{T} Q_{j}(\xi, \eta) \\
& \quad=\Psi_{z}^{i T} \boldsymbol{Q}
\end{aligned}
$$

Substituting Eqs. ( 3 ) - (8) into Eq. (1), Eq. (9) can be obtained.

$$
\begin{aligned}
& \int_{-1}^{1}\left(\left\{\boldsymbol{\Psi}_{x \zeta}^{i T}, \boldsymbol{\Psi}_{y \zeta}^{i T}, \boldsymbol{\Psi}_{z \zeta}^{i T}\right\}\left[\begin{array}{lll}
\boldsymbol{A}_{11}^{i} & \boldsymbol{A}_{12}^{i} & \boldsymbol{A}_{13}^{i} \\
\boldsymbol{A}_{21}^{i} & \boldsymbol{A}_{22}^{i} & \boldsymbol{A}_{23}^{i} \\
\boldsymbol{A}_{31}^{i} & \boldsymbol{A}_{32}^{i} & \boldsymbol{A}_{33}^{i}
\end{array}\right]\left\{\begin{array}{c}
\boldsymbol{X}_{x \zeta}^{i} \\
\boldsymbol{X}_{y \zeta}^{i} \\
\boldsymbol{X}_{z \zeta}^{i}
\end{array}\right\}\right. \\
& +\left\{\Psi_{x}^{i T}, \Psi_{y}^{i T}, \Psi_{z}^{i T}\right\}\left[\begin{array}{lll}
\boldsymbol{B}_{11}^{i} & \boldsymbol{B}_{12}^{i} & \boldsymbol{B}_{13}^{i} \\
\boldsymbol{B}_{21}^{i} & \boldsymbol{B}_{22}^{i} & \boldsymbol{B}_{23}^{i} \\
\boldsymbol{B}_{31}^{i} & \boldsymbol{B}_{32}^{i} & \boldsymbol{B}_{33}^{i}
\end{array}\right]\left\{\begin{array}{l}
\boldsymbol{X}_{x}^{i} \\
\boldsymbol{X}_{y}^{i} \\
\boldsymbol{X}_{z}^{i}
\end{array}\right\} \\
& +\left\{\Psi_{x}^{i T}, \Psi_{y}^{i T}, \Psi_{z}^{i T}\right\}\left[\begin{array}{lll}
C_{11}^{i} & C_{12}^{i} & C_{13}^{i} \\
C_{21}^{i} & C_{22}^{i} & C_{23}^{i} \\
C_{31}^{i} & C_{32}^{i} & C_{33}^{i}
\end{array}\right]^{T}\left\{\begin{array}{l}
\boldsymbol{X}_{x \zeta}^{i} \\
\boldsymbol{X}_{y \zeta}^{i} \\
\boldsymbol{X}_{z \xi}^{i}
\end{array}\right\} \\
& {\left[\begin{array}{lll}
\boldsymbol{C}_{11}^{i} & \boldsymbol{C}_{12}^{i} & \boldsymbol{C}_{13}^{i}
\end{array}\right]\left[\begin{array}{l}
\boldsymbol{X}_{x}^{i} \\
\boldsymbol{X}^{i}
\end{array}\right]} \\
& \left.+\left\{\Psi_{x \zeta}^{i T}, \Psi_{y \zeta}^{i T}, \Psi_{z \zeta}^{i T}\right\}\left[\begin{array}{lll}
C_{21}^{i} & C_{22}^{i} & C_{23}^{i} \\
C_{31}^{i} & C_{32}^{i} & C_{33}^{i}
\end{array}\right]\left\{\begin{array}{c}
\boldsymbol{X}_{y}^{i} \\
\boldsymbol{X}_{z}^{i}
\end{array}\right\}\right) d \zeta \\
& =\int_{-1}^{1}\left(\left\{\Psi_{x}^{i T}, \Psi_{y_{1}}^{i T}, \Psi_{z}^{i T}\right\}\left[\begin{array}{l}
F_{1}^{i} \\
F_{2}^{i} \\
F_{3}^{i}
\end{array}\right]\right) d \zeta \\
& +\left\{\boldsymbol{\Psi}_{x}^{i T}, \boldsymbol{\Psi}_{y}^{i T}, \boldsymbol{\Psi}_{\bar{T}}^{i T}\right\}\left[\begin{array}{l}
\boldsymbol{q}_{1}^{i} \\
\boldsymbol{q}_{2}^{i} \\
\boldsymbol{q}_{3}^{i}
\end{array}\right]+\text { Res } .
\end{aligned}
$$

On $\boldsymbol{A}, \boldsymbol{B}, \boldsymbol{C}, \boldsymbol{F}$ and $\boldsymbol{q}$, refer to Ref. (4) since they are equal to ones in the case of $3 \mathrm{D}$ elasticity problem. Res is expressed as

$$
\begin{aligned}
\operatorname{Res} & =\int_{-1}^{1} \int_{-1}^{1} \int_{-1}^{1} \frac{1}{|\boldsymbol{J}|}\left\{\boldsymbol { \Psi } _ { x } ^ { i T } \left(\Delta_{11} d \sigma_{x}^{I}+\Delta_{12} d \tau_{x y}^{I}\right.\right. \\
& \left.+\Delta_{13} d \tau_{z x}^{I}\right) \boldsymbol{Q}_{\xi} \\
& +\Psi_{x}^{i T}\left(\Delta_{21} d \sigma_{x}^{I}+\Delta_{22} d \tau_{x y}^{I}+\Delta_{23} d \tau_{z x}^{I}\right) \boldsymbol{Q}_{\eta} \\
& +\boldsymbol{\Psi}_{y}^{i T}\left(\Delta_{11} d \tau_{x y}^{I}+\Delta_{12} d \sigma_{y}^{I}+\Delta_{13} d \tau_{y z}^{I}\right) \boldsymbol{Q}_{\xi} \\
& +\boldsymbol{\Psi}_{y}^{i T}\left(\Delta_{21} d \tau_{x y}^{I}+\Delta_{22} d \sigma_{y}^{I}+\Delta_{23} d \tau_{y z}^{I}\right) \boldsymbol{Q}_{\eta} \\
& +\boldsymbol{\Psi}_{z}^{i T}\left(\Delta_{11} d \tau_{z x}^{I}+\Delta_{12} d \tau_{y z}^{I}+\Delta_{13} d \sigma_{z}^{I}\right) \boldsymbol{Q}_{\xi} \\
& +\boldsymbol{\Psi}_{z}^{i T}\left(\Delta_{21} d \tau_{z x}^{I}+\Delta_{22} d \tau_{y z}^{I}+\Delta_{23} d \sigma_{z}^{I}\right) \boldsymbol{Q}_{\eta} \\
& +\boldsymbol{\Psi}_{x \zeta}^{i T}\left(\Delta_{31} d \sigma_{x}^{I}+\Delta_{32} d \tau_{x y}^{I}+\Delta_{33} d \tau_{z x}^{I}\right) \boldsymbol{Q} \\
& +\boldsymbol{\Psi}_{y \zeta}^{i T}\left(\Delta_{31} d \tau_{x y}^{I}+\Delta_{32} d \sigma_{y}^{I}+\Delta_{33} d \tau_{y z}^{I}\right) \boldsymbol{Q} \\
& \left.+\boldsymbol{\Psi}_{z \zeta}^{i T}\left(\Delta_{31} d \tau_{z x}^{I}+\Delta_{32} d \tau_{y z}^{I}+\Delta_{33} d \sigma_{z}^{I}\right) \boldsymbol{Q}\right\}|\boldsymbol{J}| d \xi d \eta d \zeta
\end{aligned}
$$

$|\boldsymbol{J}|$ is a Jacobian, and suffix $\xi, \eta, \zeta$ denote the partial differential by each direction.

$$
\Delta=\left[\begin{array}{ccc}
y_{\eta} z_{\zeta}-y_{\zeta} z_{\eta} & -x_{\eta} z_{\zeta}+x_{\zeta} z_{\eta} & x_{\eta} y_{\zeta}-x_{\zeta} y_{\eta} \\
-y_{\xi} z_{\zeta}+y_{\zeta} z_{\xi} & x_{\xi} z_{\zeta}-x_{\zeta} z_{\xi} & -x_{\xi} y_{\zeta}+x_{\xi} y_{\xi} \\
y_{\xi} z_{\eta}-y_{\eta} z_{\xi} & -x_{\xi} z_{\eta}+x_{\eta} z_{\xi} & x_{\xi} y_{\eta}-x_{\eta} y_{\xi}
\end{array}\right],
$$

Equation (10) can be arranged as

$$
\operatorname{Res}=\int_{-1}^{1}\left[\left\{\Psi_{x}^{i T}, \Psi_{y}^{i T}, \Psi_{z}^{i T}\right\}\left[\begin{array}{c}
\boldsymbol{D}_{1}^{i} \\
\boldsymbol{D}_{2}^{i} \\
\boldsymbol{D}_{3}^{i}
\end{array}\right]\right.
$$




$$
+\left\{\boldsymbol{\Psi}_{x \zeta}^{i T}, \Psi_{z \zeta}^{i T}, \Psi_{z \zeta}^{i T}\right\}\left[\begin{array}{c}
\boldsymbol{E}_{1}^{i} \\
\boldsymbol{E}_{2}^{i} \\
\boldsymbol{E}_{3}^{i}
\end{array}\right] d \zeta .
$$

$\boldsymbol{D}$ and $\boldsymbol{E}$ are expressed as follows

$$
\begin{aligned}
\boldsymbol{D}_{1}^{i} & =\int_{-1}^{1} \int_{-1}^{1}\left\{\left(\Delta_{11} d \sigma_{x}^{I}+\Delta_{12} d \tau_{x y}^{I}+\Delta_{13} d \tau_{z x}^{I}\right) \boldsymbol{Q}_{\xi}\right. \\
& \left.+\left(\Delta_{21} d \sigma_{x}^{I}+\Delta_{22} d \tau_{x y}^{I}+\Delta_{23} d \tau_{z x}^{I}\right) \boldsymbol{Q}_{\eta}\right\} d \xi d \eta \\
\boldsymbol{D}_{2}^{i} & =\int_{-1}^{1} \int_{-1}^{1}\left\{\left(\Delta_{11} d \tau_{x y}^{I}+\Delta_{12} d \sigma_{y}^{I}+\Delta_{13} d \tau_{y z}^{I}\right) \boldsymbol{Q}_{\xi}\right. \\
& \left.+\left(\Delta_{21} d \tau_{x y}^{I}+\Delta_{22} d \sigma_{y}^{I}+\Delta_{23} d \tau_{y z}^{I}\right) \boldsymbol{Q}_{\eta}\right\} d \xi d \eta \\
\boldsymbol{D}_{3}^{i} & =\int_{-1}^{1} \int_{-1}^{1}\left\{\left(\Delta_{11} d \tau_{z x}^{I}+\Delta_{12} d \tau_{y z}^{I}+\Delta_{13} d \sigma_{z}^{I}\right) \boldsymbol{Q}_{\xi}\right. \\
& \left.+\left(\Delta_{21} d \tau_{z x}^{I}+\Delta_{22} d \tau_{y z}^{I}+\Delta_{23} d \sigma_{z}^{I}\right) \boldsymbol{Q}_{\eta}\right\} d \xi d \eta \\
\boldsymbol{E}_{1}^{i} & =\int_{-1}^{1} \int_{-1}^{1}\left(\Delta_{31} d \sigma_{x}^{I}+\Delta_{32} d \tau_{x y}^{I}+\Delta_{33} d \tau_{z x}^{I}\right) \boldsymbol{Q} d \xi d \eta \\
\boldsymbol{E}_{2}^{i}= & \int_{-1}^{1} \int_{-1}^{1}\left(\Delta_{31} d \tau_{x y}^{I}+\Delta_{32} d \sigma_{y}^{I}+\Delta_{33} d \tau_{y z}^{I}\right) \boldsymbol{Q} d \xi d \eta \\
\boldsymbol{E}_{3}^{i} & =\int_{-1}^{1} \int_{-1}^{1}\left(\Delta_{31} d \tau_{x y}^{I}+\Delta_{32} d \sigma_{y}^{I}+\Delta_{33} d \tau_{y z}^{I}\right) \boldsymbol{Q} d \xi d \eta
\end{aligned}
$$

In the whole analysis region, these are expressed by the following equation.

$$
\begin{aligned}
& \boldsymbol{X}=\sum_{i}\left\{\begin{array}{l}
\boldsymbol{X}_{x}^{i} \\
\boldsymbol{X}_{y}^{i} \\
\boldsymbol{X}_{z}^{i}
\end{array}\right\}, \Psi=\sum_{i}\left\{\begin{array}{l}
\boldsymbol{\Psi}_{x}^{i} \\
\boldsymbol{\Psi}_{y}^{i} \\
\boldsymbol{\Psi}_{z}^{i}
\end{array}\right\}, \\
& \boldsymbol{A}=\sum_{i}\left[\begin{array}{lll}
\boldsymbol{A}_{11}^{i} & \boldsymbol{A}_{12}^{i} & \boldsymbol{A}_{13}^{i} \\
\boldsymbol{A}_{21}^{i} & \boldsymbol{A}_{22}^{i} & \boldsymbol{A}_{23}^{i} \\
\boldsymbol{A}_{31}^{i} & \boldsymbol{A}_{32}^{i} & \boldsymbol{A}_{33}^{i}
\end{array}\right], \\
& \boldsymbol{B}=\sum_{i}\left[\begin{array}{lll}
\boldsymbol{B}_{11}^{i} & \boldsymbol{B}_{12}^{i} & \boldsymbol{B}_{13}^{i} \\
\boldsymbol{B}_{21}^{i} & \boldsymbol{B}_{22}^{i} & \boldsymbol{B}_{23}^{i} \\
\boldsymbol{B}_{31}^{i} & \boldsymbol{B}_{32}^{i} & \boldsymbol{B}_{33}^{i}
\end{array}\right], \\
& \boldsymbol{C}=\sum_{i}\left[\begin{array}{lll}
C_{11}^{i} & C_{12}^{i} & C_{13}^{i} \\
C_{21}^{i} & C_{22}^{i} & C_{23}^{i} \\
C_{31}^{i} & C_{32}^{i} & C_{33}^{i}
\end{array}\right], \\
& \boldsymbol{D}=\sum_{i}\left\{\begin{array}{c}
D_{1}^{i} \\
D_{2}^{i} \\
D_{3}^{i}
\end{array}\right\}, \quad \boldsymbol{E}=\sum_{i}\left\{\begin{array}{l}
\boldsymbol{E}_{1}^{i} \\
\boldsymbol{E}_{2}^{i} \\
\boldsymbol{E}_{3}^{i}
\end{array}\right\}, \\
& \boldsymbol{F}=\sum_{i}\left\{\begin{array}{l}
\boldsymbol{F}_{1}^{i} \\
\boldsymbol{F}_{2}^{i} \\
\boldsymbol{F}_{3}^{i}
\end{array}\right\}, \quad \boldsymbol{q}=\sum_{i}\left\{\begin{array}{l}
\boldsymbol{q}_{1}^{i} \\
\boldsymbol{q}_{2}^{2} \\
\boldsymbol{q}_{3}^{i}
\end{array}\right\}
\end{aligned}
$$

Therefore, the principle of Discrete Virtual Work by the 3D elastoplastic MAL can be obtained as follows.

$$
\begin{gathered}
\int_{-1}^{1}\left[\boldsymbol{\Psi}_{\zeta}^{T} \boldsymbol{A} \boldsymbol{X}_{\zeta}+\boldsymbol{\Psi}^{T} \boldsymbol{C}^{T} \boldsymbol{X}_{\zeta}+\boldsymbol{\Psi}_{\zeta}^{T} \boldsymbol{C} \boldsymbol{X}\right. \\
\left.\quad+d \boldsymbol{\Psi}^{T} \boldsymbol{B} \boldsymbol{X}-\boldsymbol{\Psi}^{T} \boldsymbol{D}-\boldsymbol{\Psi}_{\zeta}^{T} \boldsymbol{E}\right] d \zeta \\
\quad=\int_{-1}^{1} \boldsymbol{\Psi}^{T}(\zeta) \boldsymbol{F} d \zeta+\boldsymbol{\Psi}^{T}(\zeta) \boldsymbol{q}
\end{gathered}
$$

Integrating by parts the left-hand side of Eq. (11), we have

$$
\begin{aligned}
& \int_{-1}^{1} \boldsymbol{\Psi}^{T}\left\{\left[-\boldsymbol{A} \boldsymbol{X}_{\zeta}\right]^{\prime}+\boldsymbol{C}^{T} \boldsymbol{X}_{\zeta}-[\boldsymbol{C} \boldsymbol{X}]^{\prime}+\boldsymbol{B} \boldsymbol{X}\right\} \\
& \left.\quad-\boldsymbol{D}+\boldsymbol{E}_{\zeta}\right\} d \zeta \\
& \quad+\left.\left[\boldsymbol{\Psi}^{T} \boldsymbol{A} \boldsymbol{X}_{\zeta}+\boldsymbol{\Psi}^{T} \boldsymbol{C}-\boldsymbol{\Psi}^{T} \boldsymbol{E} \boldsymbol{X}\right]\right|_{-1} ^{1} \\
& \quad=\int_{-1}^{1} \boldsymbol{\Psi}^{T} \boldsymbol{F} d \zeta+\left.\boldsymbol{\Psi}^{T} \boldsymbol{q}\right|_{\zeta= \pm 1}
\end{aligned}
$$

Considering $\Psi$, which become zero at both edges ( $\zeta=$ \pm 1 ) in Eq. (12), the following equation is obtained.

$$
\begin{gathered}
\int_{-1}^{1} \boldsymbol{\Psi}^{\tau}\left\{\left[-\boldsymbol{A} \boldsymbol{X}_{\zeta}\right]^{\prime}+\boldsymbol{C}^{T} \boldsymbol{A}_{\zeta}-[\boldsymbol{C X}]^{\prime}+\boldsymbol{B} \boldsymbol{X}\right\} \\
\left.-\boldsymbol{D}+\boldsymbol{E}_{\zeta}\right\} d \zeta=\int_{-1}^{1} \boldsymbol{\Psi}^{T} \boldsymbol{F} d \zeta
\end{gathered}
$$

Since the above equation is established for all $\Psi$ except at both edges, we have

$$
\begin{aligned}
& -\boldsymbol{A} \boldsymbol{X}_{\zeta \zeta}-\left(\boldsymbol{A}_{\zeta}-\boldsymbol{C}^{T}+\boldsymbol{C}\right) \boldsymbol{X}_{\zeta}+\left(\boldsymbol{B}-\boldsymbol{C}_{\zeta}\right) \boldsymbol{X} \\
& \quad=\boldsymbol{F}+\boldsymbol{D}-\boldsymbol{E}_{\zeta}
\end{aligned}
$$

Substituting Eq. (13) into Eq. (12), the boundary condition is obtained as

$$
\boldsymbol{A} \boldsymbol{X}_{\zeta}+\boldsymbol{C X}=\boldsymbol{E} \pm\left.\boldsymbol{q}\right|_{\zeta= \pm 1}
$$

Solving Eq. (13) under Eq.(14), we can obtained displacement increment and strain increment in the optional point on the curve, and substituting them into Eq. (3) - (5), we can also obtained the strain increment and stress increment in the domain.

\section{Numerical Method}

In this work, the B-Spline collocation method is employed to solve the boundary value problem for ordinary differential equations (Eqs.(13) and (14)) derived in pre-chapter. In addition, the concept of the evaluation point is introduced as judging elastic region and plastic region. In the local coordinate system, coordinates of Gauss integration points and both edges are chosen on $\xi$ and $\eta$ direction, and the coordinates of the collocation points are chosen in the $\zeta$ direction, and the evaluation points are placed for getting by the combination of these coordinates. The arrangement of the evaluation points are shown in Fig. 2, when the number of Gauss points and collocation points are 2 and 5 , respectively. Although the arrangement of actual evaluation points is shown only in the upper surface, the evaluation points are also similarly placed with the upper surface in plane surrounded in dotted line at some $\zeta$ and bottom face. Next, the flowchart of the program developed in this study is shown in Fig. 3. The residual energy is generated in the plastic region, when Eqs. (13) and (14) are solved by the BVP-ODE solver. The values of $\boldsymbol{D}, \boldsymbol{E}$ and $\boldsymbol{E}_{\zeta}$ in Eqs. (13) and (14) change with ones before the calculation, when the residual energy is generated. Therefore, the values of $\boldsymbol{D}, \boldsymbol{E}$ and $\boldsymbol{E}_{5}$ are renewed, and Eqs. (13) and (14) are resolved again. It is considered the convergence of the calculation, when the stress error consists under the required allowable error by this iteration. When the solution converged, the load increment or displacement increment is newly given, and repeats the iteration until the convergence. The method of Miyoshi et al. ${ }^{(5)}$ is used for obtaining the stress increment from the strain increment in this work. 


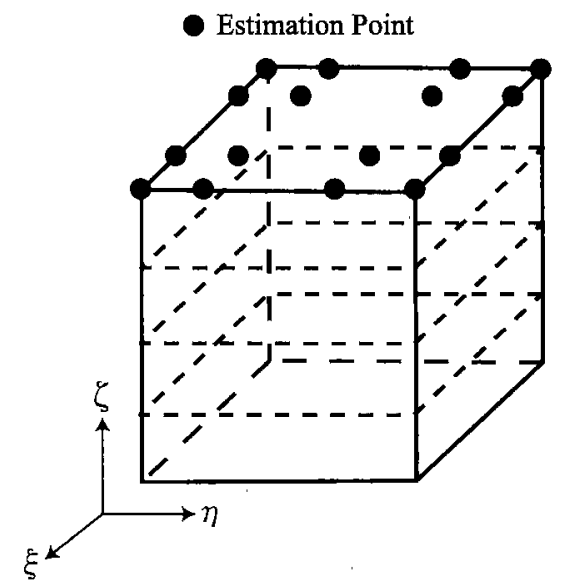

Fig. 2 Configuration of estimation point in the master element

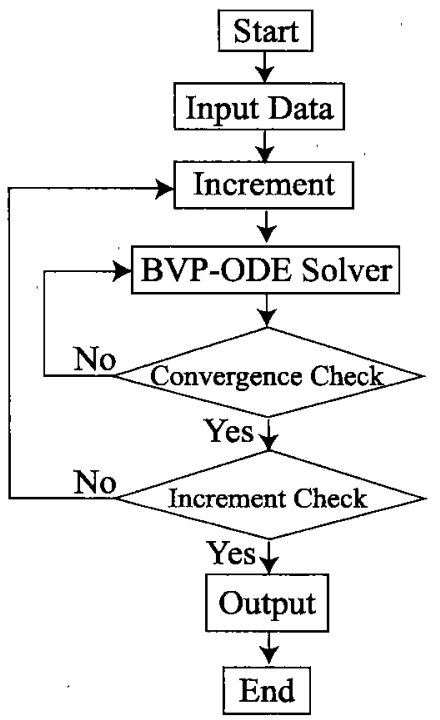

Fig. 3 Program flowchart

\section{Numerical Results and Discussion}

In order to verify the calculation accuracy and efficiency of the MAL in the 3D elastoplastic problems, BGA model which was a method of solder joints shown in Fig. 4(a) was analyzed. The bottom of the solder was restricted in the $x, y$ and $z$ direction, front surface was restricted in the $y$ direction and top surface was restricted in the $y, z$ direction. Then, an enforcement displacement $\delta=0.002 \mathrm{~mm}$ was given on the top surface in the $x$ axial direction. Young's modulus was $35 \mathrm{GPa}$, Poisson ratio was 0.3 , Yield stress was $26.4 \mathrm{MPa}$ and work hardening was 2.77 GPa.

Since it does not discrete in the $\zeta$ direction, MAL is possible to obtain the higher accuracy, when the direction of which change of stress and strain are large is made to be the $\zeta$ direction. And, it is possible

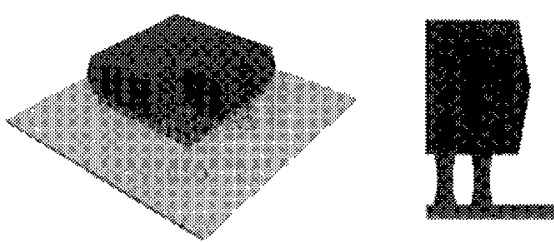

(a) Ball grid array joints

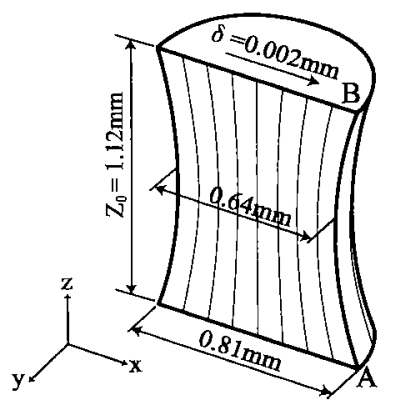

(b) MAL division number (c) of lines ; 41

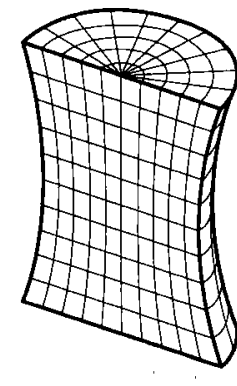

FEM division number of nodes; 2332
Fig. 4 The three-dimensional elasto-plastic analysis of BGA model

to carry out the regional division in the less curve number, when the curves are placed along the longitudinal direction. An example of element division of MAL considering above mentions is shown in Fig. 4 (b). And an example of element division of FEM is shown in Fig. 4(c).

The change rate of the stress was used in convergence decision in iterations and the relative error $e$ in the whole analytical region is defined by the following equation when the Mises' equivalent stress denotes $\bar{\sigma}_{n}^{i}$ at the evaluation point of $i(1 \leq i \leq m)$ in the $n$-th iteration.

$$
e=\sum_{i=1}^{m}\left\{\frac{\left|\bar{\sigma}_{n}^{i}-\bar{\sigma}_{n-1}^{i}\right|}{\bar{\sigma}_{n}^{i}}\right\}
$$

An example of the convergence process is shown in Fig. 5. Though the error smoothly decreases with iteration to near $10^{-9}$, and it begins to gradually vibrate when it decreases from $10^{-9}$, and it greatly vibrates near $10^{-11}$. Therefore, the allowable error is made to be $10^{-8}$ in this analysis in order to surely converge the solution. Then, it is considered the convergence of the solution, when the error consists under the allowable error.

Figure 6 shows the change of equivalent stress distributions and the extent of the plastic regions with the increase in the enforcement displacement magnitude. The upper part shows equivalent stress distributions of the whole BGA model, and the under side shows only that time plastic regions. It is shown that the plastic regions gradually spread from Fig. 6 (a) to Fig. 6(c) with the increase in enforcement displace- 
ment magnitude. From this figure, it is understood that the elastoplastic analysis can be calculated by the method proposed in this work. The MAL results of equivalent stress distributions and $\sigma_{z}$ distributions along the curve $\mathrm{AB}$ shown in Fig. 4 (b) are demonstrated in Figs. 7 and 8, respectively, in the case of 27 , 41 and 55 lines. The horizontal axis is made dimensionless by dividing by height $Z_{0}$ of the BGA model. Although the equivalent stresses can be obtained almost similar distributions without being dependent on the number of the lines, the calculation result of $\sigma_{z}$ in case of curve number of 27 has lowered in comparison with 41 or 55 lines on the $z / Z_{0}=0.0$ and $z / Z_{0}=1.0$. These results show that to some extent division is necessary in $\xi$ and $\eta$ directions, since the MAL discretization is equivalent to FEM for $\xi$ and $\eta$ directions in the local coordinate system. On this model,

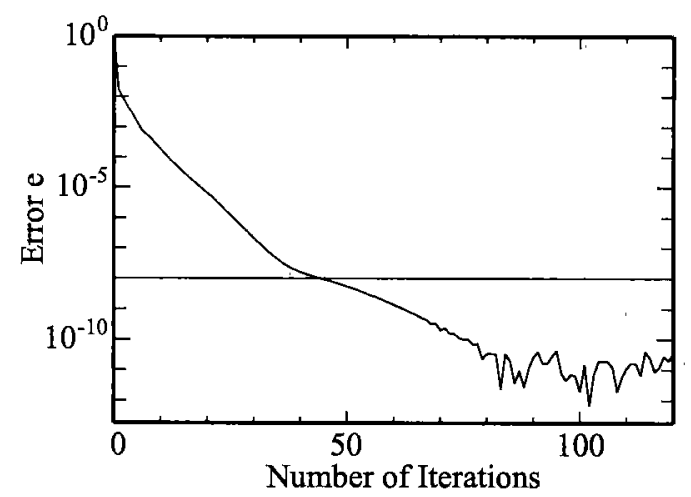

Fig. 5 The change of the error $e$ in an iterative process however, the generally similar stress distribution has been obtained, and it is understood that the analysis can be carried out at the sufficient accuracy, if the curve number is about 41 .

Next, the calculation results of MAL and FEM are compared. The distributions of $\sigma_{z}$ along the curve

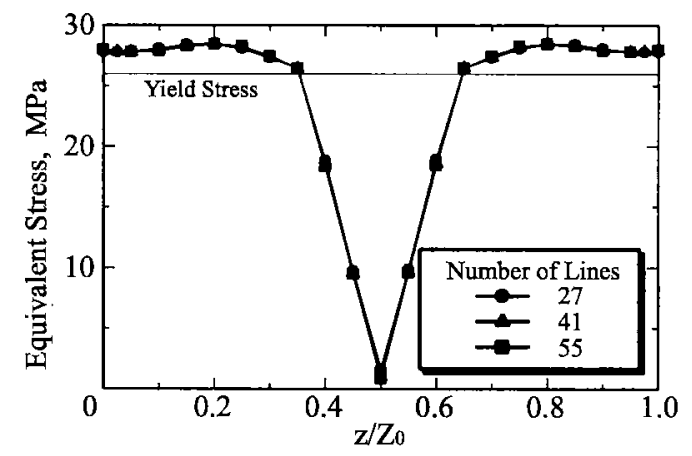

Fig. 7 Equivalent stress distributions by the MAL analysis

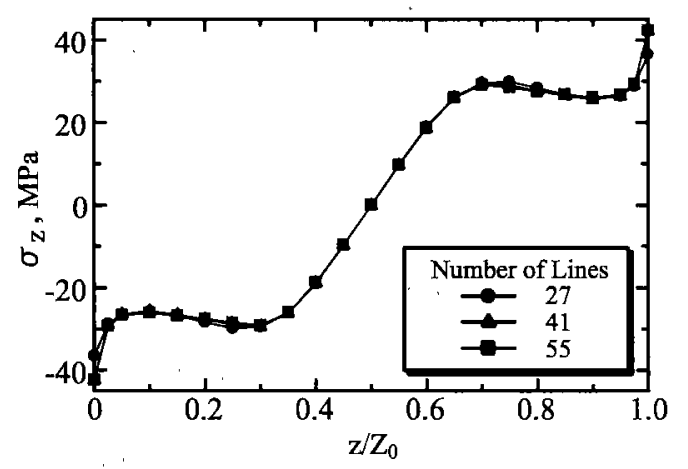

Fig. 8 . Distributions of $\sigma_{z}$ by the MAL analysis

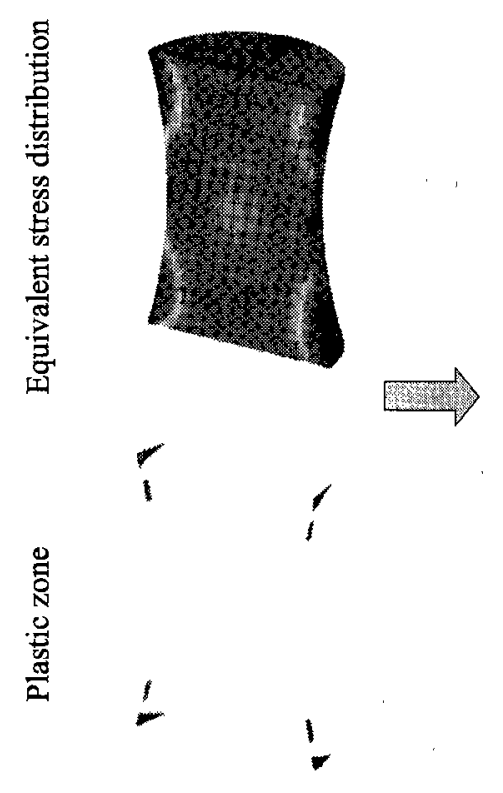

(a)
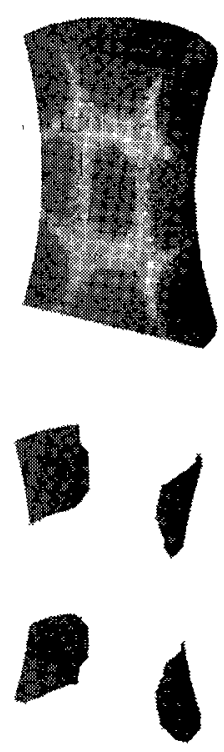

(b)

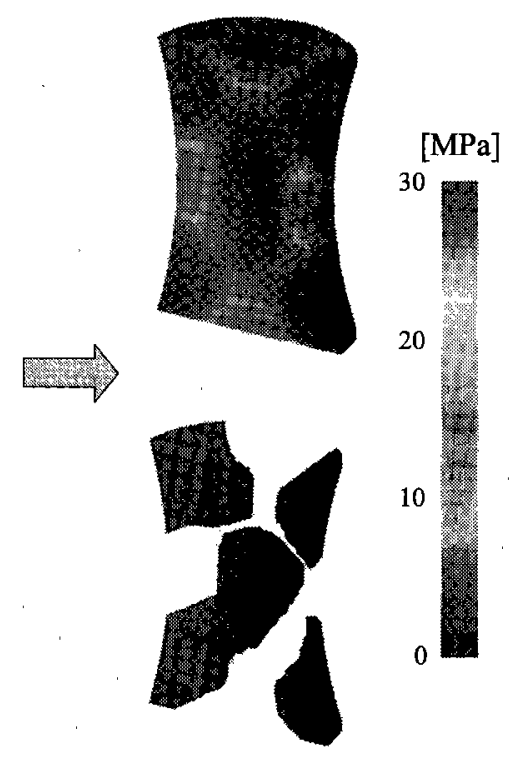

(c)

Fig. 6 Transition of the stress distribution with the change of the enforcement displacement 


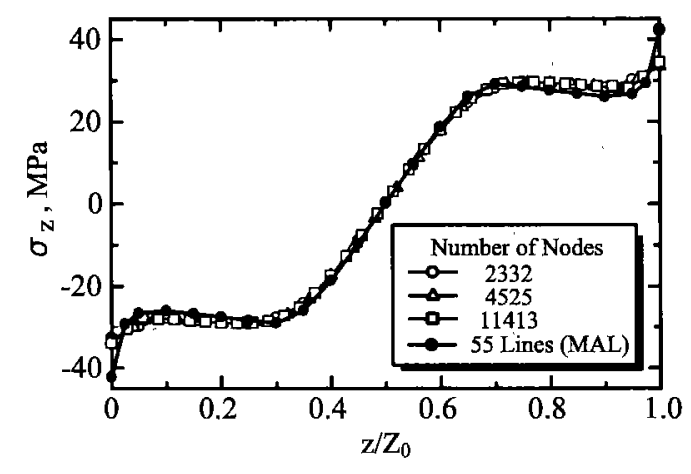

Fig. 9 Comparison of MAL results with FEM ones

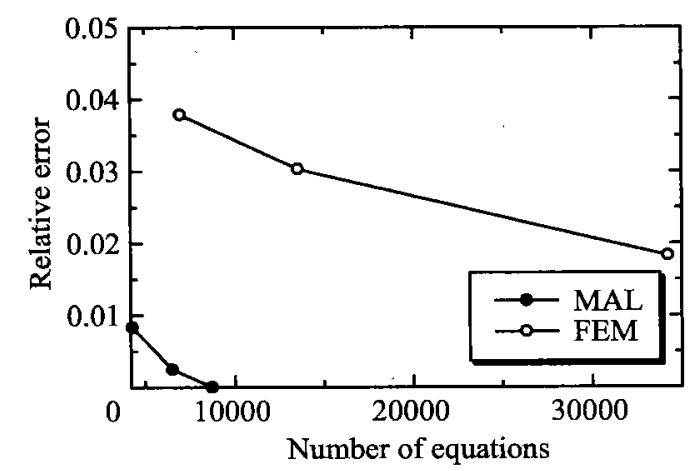

Fig. 10 Relationship between the number of simultaneous linear equations and relative error

$A B$ are shown in Fig. 9, when the number of curves is 55 in MAL and the number of nodes are 2332, 4525, 11413 in FEM. In the analysis of FEM, generalpurpose finite element analysis program MSC/NASTRAN Ver. 70.7 was used. The calculation results of MAL and FEM are similar as a whole. However, the results of FEM are lower than those of MAL when the both edges are especially noticed, and these values are lower than the value of MAL in curve number of 27 . And, the results of FEM gradually approach the results of MAL with the increase in the node number. Therefore, FEM results seem to approach the MAL results, when the node number of FEM is more increased.

Then, the relative error of equivalent stress of MAL and FEM at $z / Z_{0}=1.0$ is shown in Fig. 10. As a standard value, numerical result of MAL in 55 lines was used. The horizontal axis takes a number of simultaneous linear equations which the BVP-ODE solver has to solve in MAL, and it takes a number of whole stiffness matrix in FEM. The size of the matrix of the simultaneous linear equation is primarily determines the memory requirements and calculation time in MAL and FEM, and it is possible to carry out the calculation by the less memory in the short time, as the number of simultaneous linear equations is less. In order to accurately compare the calculation efficiency, the same computer must be used. However, the vector processor SX-4 of the Yamaguchi University integrated information processing center was used in FEM and the computer equipped with Intel Celeron $500 \mathrm{MHz} 2 \mathrm{CPU}$ was used in MAL, since the $\mathrm{C}++$ compiler used by the MAL program has not been improved in SX-4. Therefore, in making the number of simultaneous linear equations to be a parameter, the calculation efficiency was compared. The relative errors of A and B decrease with the increase in simultaneous linear equation numbers as shown in Fig. 10. The relative error of MAL is more located under the left than that of FEM in Fig. 10, and it is seen that the MAL solution has high accuracy compared with the FEM one in spite of the less equation number. Therefore, it is considered that the MAL is an effective method for three-dimensional elastoplastic stress analysis.

\section{Conclusions}

By expressing the displacement increment and the virtual displacement increment as continuous functions in some unidirection, the principle of discrete virtual work on arbitrary lines based on the iterative method was derived from the principle of the three-dimensional increment virtual work, and it finally was made to result in the boundary value problem of simultaneous ordinary differential equations. In addition, the program was developed on the basis of this theory, and the three-dimensional elastoplastic analysis of the BGA model was carried out. As a result, it was confirmed that high accuracy was obtained stedily and to solve the three-dimensional elastoplastic problem was possible by the method of arbitrary lines.

\section{References}

(1) Kaminishi, K. and Miyoshi, T., Proc. HERMIS '94, (1994), pp. 236-245.

(.2) Kaminishi, K. and Miyoshi, T., Proc. ICES '95, (1995), pp. 1298-1303.

(3) Kaminishi, K., Trans. Jpn. Soc. Mech. Eng., (in Japanese), Vol.61, No.592, A (1995), pp. 2677 2683.

(4) Kaminishi, K. and Ando, R., Trans Jpn. Soc. Mech., Vol. 66, No. 644, A (2000), pp. 685-691.

(5) Miyoshi, T., Kaminishi, K. and Kawano, S., Comp. Mech., 3 (1988), pp. 371-379. 